\title{
Periodicity and ergodicity for abstract evolution equations with critical nonlinearities
}

\author{
Bruno de Andrade ${ }^{1}$, Claudio Cuevas ${ }^{2 *}$, Jin Liang ${ }^{3}$ and Herme Soto ${ }^{4}$
}

\section{"Correspondence:}

cch@dmat.ufpe.br

${ }^{2}$ Departamento de Matemática,

Universidade Federal

de Pernambuco, Recife,

PE 50540-740, Brazil

Full list of author information is

available at the end of the article

\begin{abstract}
We study the existence of pseudo almost periodic mild solutions for the abstract evolution equation $u^{\prime}(t)=A u(t)+f(t, u(t)), t \in \mathbb{R}$, when the nonlinearity $f$ satisfies certain critical conditions. We apply our abstract results to the heat equation.

MSC: Primary 35B15; secondary 35K05; 58J35
\end{abstract}

Keywords: pseudo almost periodic solutions; critical nonlinearities; growth conditions

\section{Introduction}

We observe that real systems usually exhibit internal variations or are submitted to external perturbations. In many situations we can assume that these variations are approximately periodic in a broad sense. In the literature several concepts have been studied to represent the idea of approximately periodic function (see [1]). Most of the works deal with asymptotically periodic functions and almost periodic functions (e.g., [2, 3]). As a natural generalization of the classical almost periodicity in the sense of Bochner, the concept of pseudo almost periodicity as well as its various extensions have been studied extensively by many researchers in the last 20 years (cf., e.g., [4-14]). Pseudo almost periodic functions have many applications in several problems, for example in the theory of functional differential equations, integral equations, and partial differential equations. The concept of pseudo almost periodicity, which is the central issue in this work, was introduced by Zhang [15-18] in the early 1990s. Our purpose in this work is to analyze the existence of pseudo almost periodic mild solutions of the abstract problem

$$
u^{\prime}(t)=A u(t)+f(t, u(t)), \quad t \in \mathbb{R},
$$

where the linear operator $A: D(A) \subset X^{0} \rightarrow X^{0}$ is such that $-A$ is a sectorial operator in the Banach space $X^{0}$ and $f$ is a continuous function. Many authors have considered abstract linear evolution equations in Banach spaces (see [19-23] and references therein). In particular several results on the existence of pseudo almost periodic solutions to differential equations of the type (1.1) have been established. Stimulated by these works, in this paper, we will investigate further the corresponding problem where the nonlinearity of the nonlinear term of (1.1) has critical growth. Note that there is much interest in the

(c) 2015 de Andrade et al.; licensee Springer. This is an Open Access article distributed under the terms of the Creative Commons Attribution License (http://creativecommons.org/licenses/by/4.0), which permits unrestricted use, distribution, and reproduction in any medium, provided the original work is properly credited. 
study of nonlinearities when they are assumed to be a critical cause and intrinsic difficult. We need to understand the scale of fractional powers spaces associated to the linear operator $A$, especially the embeddings into known spaces like $L^{p}$ spaces, and the $\epsilon$-regularity properties (see Definition 2.4) of the nonlinearity $f$ in this scale of spaces. It makes the analysis much more technical and harder. Problems with this kind of nonlinearity appear frequently in the heat equation theory. However, to the best of our knowledge, there are few results for these problems.

We will denote by $X^{\beta}:=\left(D\left(A^{\beta}\right),\|\cdot\|_{X^{\beta}}\right), \beta \geq 0$, the fractional power spaces associated to the operator $A$ and by $\left\{e^{A t}\right\}_{t \geq 0}$ the analytic semigroup generated by $A$. Furthermore, we will suppose that $\operatorname{Re} \sigma(-A)>a>0$. Consequently, there exists a positive constant $M$ such that $e^{A t}$ verifies

$$
\left\|e^{A t} x\right\|_{X^{\beta}} \leq M t^{-\beta} e^{-a t}\|x\|_{X^{0}}, \quad t>0, \beta \geq 0 .
$$

The main scope of this work is to provide sufficient condition to ensure the existence of a pseudo almost periodic $\epsilon$-regular mild solution of (1.1). By an $\epsilon$-regular mild solution of (1.1) we understand a continuous function $u: \mathbb{R} \rightarrow X^{1}$ such that $u \in C\left(\mathbb{R}, X^{1+\epsilon}\right)$ and verifies

$$
u(t)=\int_{-\infty}^{t} e^{A(t-s)} f(s, u(s)) d s, \quad t \in \mathbb{R} .
$$

The notion of $\epsilon$-regular mild solution for abstract semilinear evolution equations was introduced by Arrieta and Carvalho in [24] (see also [25]) where a detailed discussion was presented considering the importance of these types of solutions.

A typical example that we will treat in this work is to consider in $L^{2}(\Omega)$, with $\Omega \subset \mathbb{R}^{3}$ a bounded smooth domain, the heat equation

$$
\begin{cases}u_{t}=A u+g h(u), & \text { in } \mathbb{R} \times \Omega, \\ u=0, & \text { on } \mathbb{R} \times \partial \Omega,\end{cases}
$$

where $g: \mathbb{R} \rightarrow \mathbb{R}$ is a pseudo almost periodic function, the function $h: \mathbb{R} \rightarrow \mathbb{R}$ verifies $|h(t)-h(s)| \leq c\left(1+|t|^{4 / 3}+|s|^{4 / 3}\right)|t-s|$, with $h(0) \neq 0$, and the operator $A u=\Delta u-a u, a>0$, is considered with domain

$$
D(A):=H^{2}(\Omega) \cap H_{0}^{1}(\Omega) .
$$

Let $L=\Delta$ with Dirichlet boundary conditions in $\Omega$. Then $L$ can be seen as an unbounded operator in $E_{2}^{0}=L^{2}(\Omega)$ with domain $D(A)$. The scale of the fractional power spaces $\left\{E_{2}^{\alpha}\right\}_{\alpha \in \mathbb{R}}$ associated to $L$ verifies (see $[24,26]$ )

$$
E_{2}^{\alpha} \hookrightarrow H^{2 \alpha}(\Omega), \quad \alpha \geq 0, \quad E_{2}^{-\alpha}=\left(E_{2}^{\alpha}\right)^{\prime}, \quad \alpha \geq 0 .
$$

Therefore, we get

$$
\begin{aligned}
& E_{2}^{\alpha} \hookrightarrow L^{r}(\Omega), \quad \text { for } r \leq \frac{6}{3-4 \alpha}, 0 \leq \alpha<\frac{3}{4}, \\
& E_{2}^{0}=L^{2}(\Omega), \\
& E_{2}^{\alpha} \hookleftarrow L^{s}(\Omega), \quad \text { for } s \geq \frac{6}{3-4 \alpha},-\frac{3}{4}<\alpha \leq 0 .
\end{aligned}
$$


Let $L_{\alpha}$ be the realization of $L$ in $E_{2}^{\alpha}$. Then we find that $L_{\alpha}$ is an isometry from $E_{2}^{\alpha+1}$ into $E_{2}^{\alpha}$ and

$$
L_{\alpha}: D\left(L_{\alpha}\right)=E_{2}^{\alpha+1} \subset E_{2}^{\alpha} \rightarrow E_{2}^{\alpha}
$$

is a sectorial operator. Denote $X_{2}^{\alpha}:=E_{2}^{\alpha-1}, \alpha \in \mathbb{R}$. The fractional power spaces associated to $L_{-1}: X_{2}^{1} \subset X_{2}^{0} \rightarrow X_{2}^{0}$ satisfy

$$
\begin{aligned}
& X_{2}^{\alpha} \hookrightarrow L^{r}(\Omega), \quad \text { for } r \leq \frac{6}{7-4 \alpha}, 1 \leq \alpha<\frac{7}{4}, \\
& X_{2}^{1}=L^{2}(\Omega), \\
& X_{2}^{\alpha} \hookleftarrow L^{s}(\Omega), \quad \text { for } s \geq \frac{6}{7-4 \alpha}, \frac{1}{4}<\alpha \leq 1 .
\end{aligned}
$$

To rewrite (1.3) in the abstract form (1.1) consider the function

$$
f(t, \psi)(x)=g(t) h(\psi(x))
$$

It follows from Section 4 , for $t \in \mathbb{R}$ fixed, that the function $f(t, \cdot)$ is an ultra-critical $\epsilon$-regular map relative to the pair $\left(X_{2}^{0}, X_{2}^{1}\right)$, for every $\epsilon \in\left(0, \frac{3}{7}\right)$. In fact, we see, for each $t \in \mathbb{R}$, that

$$
f(t, \cdot): X_{2}^{1+\epsilon} \rightarrow X_{2}^{\frac{7 \epsilon}{3}}
$$

is well defined and satisfies

$$
\|f(t, u)-f(t, v)\|_{X_{2}^{\frac{7 \epsilon}{3}}} \leq c\|g\|_{\infty}\left(1+\|u\|_{X_{2}^{1+\epsilon}}^{4 / 3}+\|v\|_{X_{2}^{1+\epsilon}}^{4 / 3}\right)\|u-v\|_{X_{2}^{1+\epsilon}},
$$

for some $c>0$. Furthermore $f$ is a pseudo almost periodic function (see Definition 2.3). For $\epsilon \in\left(0, \frac{3}{7}\right)$, it follows from Theorem 3.1 that if $\|g\|_{\infty}$ is small enough then (1.3) has a pseudo almost periodic mild solution $u: \mathbb{R} \rightarrow X^{1+\epsilon}$. This solution is given by

$$
u(t, x)=\int_{-\infty}^{t} e^{A(t-s)} g(s) h(u(s, x)) d s, \quad t \in \mathbb{R}, x \in \Omega .
$$

Furthermore, $u \in \operatorname{PAP}\left(X^{1+\theta}\right)$, for every $0 \leq \theta<\frac{7 \epsilon}{3}$.

In Section 4 we will treat in the $L^{q}$ setting the general situation,

$$
\begin{cases}u_{t}=A u+g h(u), & \text { in } \mathbb{R} \times \Omega, \\ u=0, & \text { on } \mathbb{R} \times \partial \Omega,\end{cases}
$$

where $g: \mathbb{R} \rightarrow \mathbb{R}$ is a pseudo almost periodic function, $A u=\Delta u-a u, a>0$, and the function $h: \mathbb{R} \rightarrow \mathbb{R}$ verifies

$$
|h(x)-h(y)| \leq c\left(1+|x|^{\rho-1}+|y|^{\rho-1}\right)|x-y|, \quad \rho>1,
$$

for some $c>0$. 


\section{Background material}

Consider Banach spaces, $Y$ and $Z$, with norms $\|\cdot\|_{Y}$ and $\|\cdot\|_{Z}$, respectively. Let $\operatorname{AP}(Y)$ be the set of all almost periodic functions, that is, the set of all continuous functions $g: \mathbb{R} \rightarrow Y$ such that for all $\alpha>0$ there exists $l(\alpha)>0$ such that every interval $I \subset \mathbb{R}$ of length $l(\alpha)$ contains a number $\tau$ with the property that

$$
\|g(t+\tau)-g(t)\|_{Y}<\alpha, \quad \forall t \in \mathbb{R}
$$

Also, let $P_{0}(Y)$ be the set of all bounded continuous functions $\phi: \mathbb{R} \rightarrow Y$ such that

$$
\lim _{T \rightarrow \infty} \frac{1}{2 T} \int_{-T}^{T}\|\phi(t)\|_{Y} d t=0
$$

Definition 2.1 A continuous function $g: \mathbb{R} \rightarrow Y$ is called pseudo almost periodic if $g=$ $h+\phi$, where $h \in \operatorname{AP}(Y)$ and $\phi \in P_{0}(Y)$.

The functions $h$ and $\phi$ are called, respectively, the almost periodic component and the ergodic perturbation of the function $g$. As usual, we represent by $\operatorname{PAP}(Y)$ the set of all pseudo almost periodic functions $h: \mathbb{R} \rightarrow Y$. We remark that this set provided with the uniform convergence norm $\|\cdot\|_{\infty}$ is a Banach space. In fact, we find that $\left(\operatorname{PAP}(Y),\|\cdot\|_{\infty}\right)$ is a closed subset of the Banach space of bounded continuous functions.

In this work we will need a special notion of pseudo almost periodic functions. To this end, we represent by $\operatorname{AP}(Y, Z)$ the set of all continuous function $h: \mathbb{R} \times Y \rightarrow Z$ such that $h(\cdot, x)$ is an almost periodic function uniformly on bounded sets of $Y$, that is, for all $\alpha>0$ and any bounded subset $K$ of $Y$ there exists $l(\alpha)>0$ such that all interval of length $l(\alpha)$ contains a number $\tau$ with the property that

$$
\|h(t+\tau, x)-h(t, x)\|_{Z} \leq \alpha
$$

for all $t \in \mathbb{R}$ and $x \in K$.

The following lemma will be very useful in this work.

Lemma 2.2 ([27]) If $f \in \mathrm{AP}(Y ; Z)$ and $h \in \mathrm{AP}(Y)$, then the function $f(\cdot, h(\cdot)) \in \mathrm{AP}(Z)$.

Likewise, we represent by $P_{0}(Y, Z)$ the set of all continuous functions $\phi: \mathbb{R} \times Y \rightarrow Z$ such that $\phi(\cdot, x): \mathbb{R} \rightarrow Z$ is a bounded function for all $x \in Y$ and

$$
\lim _{T \rightarrow \infty} \frac{1}{2 T} \int_{-T}^{T}\|\phi(t, x)\|_{Z} d t=0
$$

uniformly in $x \in Y$.

Definition 2.3 A continuous function $g: \mathbb{R} \times Y \rightarrow Z$ is called pseudo almost periodic if $g=h+\phi$, where $g \in \operatorname{AP}(Y, Z)$ and $\phi \in P_{0}(Y, Z)$.

We represent by $\operatorname{PAP}(Y, Z)$ the set of all pseudo almost periodic functions $g: \mathbb{R} \times Y \rightarrow Z$. We finish this section with the definition of $\epsilon$-regular map. 
Definition 2.4 For $\epsilon>0$ we say that a map $g$ is an $\epsilon$-regular map relative to the pair $\left(X^{1}, X^{0}\right)$ if there exist $\rho>1, \gamma(\epsilon)$ with $\rho \epsilon \leq \gamma(\epsilon)<1$, and a positive constant $c$, such that $g: X^{1+\epsilon} \rightarrow X^{\gamma(\epsilon)}$ and

$$
\|g(x)-g(y)\|_{X^{\gamma}(\epsilon)} \leq c\left(1+\|x\|_{X^{1+\epsilon}}^{\rho-1}+\|y\|_{X^{1+\epsilon}}^{\rho-1}\right)\|x-y\|_{X^{1+\epsilon}},
$$

for all $x, y \in X^{1+\epsilon}$.

The main result of this work basically says that if $f(t, \cdot)$ is an $\epsilon$-regular map relative to the pair $\left(X^{1}, X^{0}\right)$ and belongs to $\operatorname{PAP}\left(X^{1+\epsilon}, X^{\gamma(\epsilon)}\right)$ then we will have existence of a pseudo almost periodic $\epsilon$-regular mild solution to (1.1). Furthermore this solution belongs to $\operatorname{PAP}\left(X^{1+\theta}\right)$ for all $0 \leq \theta<\gamma(\epsilon)$. It is important to remark that we obtain an existence result in $X^{1}$ without the nonlinearity being defined on $X^{1}$.

\section{Abstract results}

We start this section observing that from (1.2) follows the estimate

$$
t^{1+\theta-\beta}\left\|e^{A t} x\right\|_{X^{1+\theta}} \leq M e^{-a t}\|x\|_{X^{\beta}}, \quad 0 \leq \beta \leq 1+\theta \leq 2 .
$$

This estimate will be very useful for our purpose. We recommend to the reader the book [28] for more details and information on sectorial operators and fractional power spaces.

In this work we consider the following class of nonlinearities: with $\epsilon, \gamma(\epsilon), \rho$, and $c$ positive constants, define

$$
\mathcal{F}(\epsilon, \rho, \gamma(\epsilon), c)
$$

as the family of functions $f$ such that, for $t \in \mathbb{R}, f(t, \cdot)$ is an $\epsilon$-regular map relative to the pair $\left(X^{1}, X^{0}\right)$, satisfying

$$
\begin{aligned}
& \|f(t, x)-f(t, y)\|_{X^{\gamma}(\epsilon)} \leq c\left(1+\|x\|_{X^{1+\epsilon}}^{\rho-1}+\|y\|_{X^{1+\epsilon}}^{\rho-1}\right)\|x-y\|_{X^{1+\epsilon}}, \\
& \|f(t, x)\|_{X^{\gamma}(\epsilon)} \leq c\left(1+\|x\|_{X^{1+\epsilon}}^{\rho}\right)
\end{aligned}
$$

for all $x, y \in X^{1+\epsilon}$.

The following theorem is the main result of this work.

Theorem 3.1 Consider $f \in \mathcal{F}(\epsilon, \gamma(\epsilon), \rho, c) \cap \operatorname{PAP}\left(X^{1+\epsilon}, X^{\gamma(\epsilon)}\right)$. If the constant $c$ is small enough then the problem (1.1) has a pseudo almost periodic $\epsilon$-regular mild solution. Furthermore, this solution verifies $u \in \operatorname{PAP}\left(X^{1+\theta}\right)$, for every $0 \leq \theta<\gamma(\epsilon)$.

Proof Fix $r>0$ and let $L(r)=\max \left\{r^{-1}+r^{\rho-1}, 1+2 r^{\rho-1}\right\}$. We claim that the result is true if

$$
M c a^{\epsilon-\gamma(\epsilon)} \Gamma(\gamma(\epsilon)-\epsilon) L(r)<1 .
$$

In fact, let $B(r)$ be the closed ball

$$
B(r):=\left\{w \in \operatorname{PAP}\left(X^{1+\epsilon}\right): \sup _{t \in \mathbb{R}}\|w(t)\|_{X^{1+\epsilon}} \leq r\right\} .
$$


Define on $B(r)$ the operator $T$ by

$$
(T u)(t)=\int_{-\infty}^{t} e^{A(t-s)} f(s, u(s)) d s
$$

The set $B(r)$ is $T$-invariant. We divide the proof of this claim in two steps.

Step 1: If $u \in \operatorname{PAP}\left(X^{1+\epsilon}\right)$ then $T u \in \operatorname{PAP}\left(X^{1+\theta}\right)$, for every $0 \leq \theta<\gamma(\epsilon)$. First of all, we show that the function

$$
t \mapsto f(t, u(t))
$$

belongs to $\operatorname{PAP}\left(X^{\gamma(\epsilon)}\right)$. In fact, since $f \in \operatorname{PAP}\left(X^{1+\epsilon}, X^{\gamma(\epsilon)}\right)$ there exist $g \in \operatorname{AP}\left(X^{1+\epsilon}, X^{\gamma(\epsilon)}\right)$ and $\phi \in P_{0}\left(X^{1+\epsilon}, X^{\gamma(\epsilon)}\right)$ such that $f=g+\phi$. In the same way, $u=h+\psi$ with $h \in \operatorname{AP}\left(X^{1+\epsilon}\right)$ and $\psi \in P_{0}\left(X^{1+\epsilon}\right)$. Hence,

$$
\begin{aligned}
f(\cdot, u(\cdot)) & =g(\cdot, h(\cdot))+f(\cdot, u(\cdot))-g(\cdot, h(\cdot)) \\
& =g(\cdot, h(\cdot))+f(\cdot, u(\cdot))-f(\cdot, h(\cdot))+\phi(\cdot, h(\cdot)) .
\end{aligned}
$$

Since $g(\cdot, h(\cdot)) \in \operatorname{AP}\left(X^{\gamma(\epsilon)}\right)$, see Lemma 2.2, it remains to show that the function

$$
t \mapsto f(t, u(t))-f(t, h(t))+\phi(t, h(t)):=F(t)+\phi(t, h(t))
$$

belongs to $P_{0}\left(X^{\gamma(\epsilon)}\right)$. To this end, note that

$$
\begin{aligned}
\frac{1}{2 T} \int_{-T}^{T}\|F(t)\|_{X^{\gamma(\epsilon)}} d t \\
\leq \frac{c}{2 T} \int_{-T}^{T}\|u(t)-h(t)\|_{X^{1+\epsilon}} d t \\
\quad+\frac{c}{2 T} \int_{-T}^{T}\|u(t)\|_{X^{1+\epsilon}}^{\rho-1}\|u(t)-h(t)\|_{X^{1+\epsilon}} d t \\
\quad+\frac{c}{2 T} \int_{-T}^{T}\|h(t)\|_{X^{1+\epsilon}}^{\rho-1}\|u(t)-h(t)\|_{X^{1+\epsilon}} d t \\
\leq c\left(1+\sup _{t \in \mathbb{R}}\|u(t)\|_{X^{1+\epsilon}}^{\rho-1}+\sup _{t \in \mathbb{R}}\|h(t)\|_{X^{1+\epsilon}}^{\rho-1}\right) \frac{1}{2 T} \int_{-T}^{T}\|\psi(t)\|_{X^{1+\epsilon}} d t .
\end{aligned}
$$

Thus, $F \in P_{0}\left(X^{\gamma(\epsilon)}\right)$. On the other hand, since $h(\mathbb{R})$ is relatively compact in $X^{1+\epsilon}$, for $\alpha>0$ we can find a finite number of open balls $O_{k}$ with center $x_{k} \in h(\mathbb{R})$ and radius less than $\alpha$ such that $h(\mathbb{R}) \subset \bigcup_{k=1}^{m} O_{k}$. Clearly, we can write $\mathbb{R}=\bigcup_{k=1}^{m} B_{k}$, where $B_{k}=h^{-1}\left(O_{k}\right)$ are open subsets. Consider

$$
E_{k}:= \begin{cases}B_{1}, & k=1, \\ B_{k} \backslash \bigcup_{i=1}^{k-1} B_{i}, & k>1,\end{cases}
$$

then $E_{i} \cap E_{j}=\emptyset$, if $i \neq j$. Let $T_{0}>0$ be such that

$$
\frac{1}{2 T} \int_{-T}^{T}\left\|\phi\left(t, x_{k}\right)\right\|_{X^{\gamma}(\epsilon)} d t \leq \alpha
$$


for all $T \geq T_{0}$ and $k \in\{1, \ldots, m\}$. Furthermore, since $g \in \operatorname{AP}\left(X^{1+\epsilon}, X^{\gamma(\epsilon)}\right)$ is uniformly continuous in $x \in \overline{h(\mathbb{R})}$, one can obtain

$$
\left\|g\left(t, x_{k}\right)-g(t, x)\right\|_{X^{\gamma}(\epsilon)} \leq \alpha,
$$

for $x \in O_{k}$ and $k \in\{1, \ldots, m\}$ uniformly in $t \in \mathbb{R}$. For $T$ sufficient large and $F_{k}:=E_{k} \cap$ $[-T, T]$, we have

$$
\begin{aligned}
& \frac{1}{2 T} \sum_{k=1}^{m} \int_{F_{k}}\|\phi(t, h(t))\|_{X^{\gamma}(\epsilon)} d t \\
& \leq \frac{1}{2 T} \sum_{k=1}^{m} \int_{F_{k}}\left\|\phi(t, h(t))-\phi\left(t, x_{k}\right)\right\|_{X^{\gamma}(\epsilon)}+\left\|\phi\left(t, x_{k}\right)\right\|_{X^{\gamma}(\epsilon)} d t \\
& \leq \frac{1}{2 T} \sum_{k=1}^{m} \int_{F_{k}}\left\|f(t, h(t))-f\left(t, x_{k}\right)\right\|_{X^{\gamma}(\epsilon)} d t \\
& \quad+\frac{1}{2 T} \sum_{k=1}^{m} \int_{F_{k}}\left\|g(t, h(t))-g\left(t, x_{k}\right)\right\|_{X^{\gamma}(\epsilon)} d t \\
& \quad+\frac{1}{2 T} \sum_{k=1}^{m} \int_{F_{k}}\left\|\phi\left(t, x_{k}\right)\right\|_{X \gamma(\epsilon)} d t .
\end{aligned}
$$

Since, for $t \in F_{k}, h(t) \in O_{k}$ we have

$$
\begin{aligned}
\frac{1}{2 T} \sum_{k=1}^{m} \int_{F_{k}}\left\|f(t, h(t))-f\left(t, x_{k}\right)\right\|_{X \gamma(\epsilon)} d t \leq & c m\left(1+\sup _{t \in \mathbb{R}}\|h(t)\|_{X^{1+\epsilon}}^{\rho-1}\right) \alpha \\
& +c m\left(\max \left\|x_{k}\right\|_{X^{1+\epsilon}}\right) \alpha .
\end{aligned}
$$

Hence, from (3.3)-(3.6) it follows that $\phi(\cdot, h(\cdot)) \in P_{0}\left(X^{\gamma(\epsilon)}\right)$ and consequently $f(\cdot, u(\cdot)) \in$ $\operatorname{PAP}\left(X^{\gamma(\epsilon)}\right)$.

Now, it is clear that $T u: \mathbb{R} \rightarrow X^{1+\theta}$ is a bounded continuous function, indeed if $t_{1}, t_{2} \in \mathbb{R}$, $t_{2}>t_{1}$, then

$$
\begin{aligned}
\left\|(T u)\left(t_{2}\right)-(T u)\left(t_{1}\right)\right\|_{X^{1+\theta}} \leq & \left\|\left(e^{A\left(t_{2}-t_{1}\right)}-I\right) \int_{-\infty}^{t_{1}} e^{A\left(t_{1}-s\right)} f(s, u(s)) d s\right\|_{X^{1+\theta}} \\
& +\left\|\int_{t_{1}}^{t_{2}} e^{A\left(t_{2}-s\right)} f(s, u(s)) d s\right\|_{X^{1+\theta}} \cdot
\end{aligned}
$$

In the above inequality, the first term clearly goes to zero as $t_{2} \rightarrow t_{1}^{+}$. For the second term we have

$$
\begin{aligned}
& \left\|\int_{t_{1}}^{t_{2}} e^{A\left(t_{2}-s\right)} f(s, u(s)) d s\right\|_{X^{1+\theta}} \\
& \quad \leq M \int_{t_{1}}^{t_{2}}\left(t_{2}-s\right)^{-1+\gamma(\epsilon)-\theta} e^{-a\left(t_{2}-s\right)}\|f(s, u(s))\|_{X^{\gamma}(\epsilon)} d s \\
& \quad \leq M a^{\theta-\gamma(\epsilon)} \sup _{s \in \mathbb{R}}\|f(s, u(s))\|_{X^{\gamma}(\epsilon)} \int_{0}^{a\left(t_{2}-t_{1}\right)} s^{-1+\gamma(\epsilon)-\theta} e^{-s} d s,
\end{aligned}
$$


which goes to zero as $t_{2} \rightarrow t_{1}^{+}$. The case $t_{1}>t_{2}$ is similar. Furthermore,

$$
\sup _{t \in \mathbb{R}}\|(T u)(t)\|_{X^{1+\theta}} \leq M a^{\theta-\gamma(\epsilon)} \Gamma(\gamma(\epsilon)-\theta) \sup _{t \in \mathbb{R}}\|f(t, u(t))\|_{X^{\gamma(\epsilon)}} .
$$

On the other hand, consider, $g_{1} \in \operatorname{AP}\left(X^{\gamma(\epsilon)}\right)$ and $\phi_{1} \in P_{0}\left(X^{\gamma(\epsilon)}\right)$ such that $f(t, u(t))=g_{1}(t)+$ $\phi_{1}(t), t \in \mathbb{R}$. Then

$$
(T u)(t)=\int_{-\infty}^{t} e^{A(t-s)} g_{1}(s) d s+\int_{-\infty}^{t} e^{A(t-s)} \phi_{1}(s) d s:=G(t)+\Phi(t), \quad t \in \mathbb{R} .
$$

To complete the proof of the Step 1, it remains to show that $G \in \operatorname{AP}\left(X^{1+\theta}\right)$ and $\Phi \in$ $P_{0}\left(X^{1+\theta}\right)$, for every $0 \leq \theta<\gamma(\epsilon)$. To this end, given $\alpha>0$, consider $l(\alpha)>0$ such that every interval of length $l(\alpha)$ contains a number $\tau$ such that

$$
\left\|g_{1}(t+\tau)-g_{1}(t)\right\|_{X \gamma(\epsilon)} \leq \frac{\alpha}{M a^{\theta-\gamma(\epsilon)} \Gamma(\gamma(\epsilon)-\theta)},
$$

for each $t \in \mathbb{R}$. Then the estimate

$$
\|G(t+\tau)-G(t)\|_{X^{1+\theta}} \leq M \int_{0}^{\infty} s^{-1+\gamma(\epsilon)-\theta} e^{-a s}\left\|g_{1}(t-s-\tau)-g_{1}(t-s)\right\|_{X \gamma(\epsilon)} d s \leq \alpha
$$

is responsible for the fact that $G \in \operatorname{AP}\left(X^{1+\theta}\right)$, for every $0 \leq \theta<\gamma(\epsilon)$. Finally, for $T>0$ we see that

$$
\begin{aligned}
\frac{1}{2 T} \int_{-T}^{T}\|\Phi(t)\|_{X^{1+\theta}} d t & \leq \frac{1}{2 T} \int_{-T}^{T} \int_{0}^{\infty}\left\|e^{A s} \phi_{1}(t-s)\right\|_{X^{1+\theta}} d s d t \\
& \leq M \int_{0}^{\infty} s^{-1+\gamma(\epsilon)-\theta} e^{-a s} \phi_{T}(s) d s,
\end{aligned}
$$

where

$$
\Phi_{T}(s)=\frac{1}{2 T} \int_{-T}^{T}\left\|\phi_{1}(t-s)\right\|_{X \gamma(\epsilon)} d t, \quad s \geq 0 .
$$

It is not hard to check that

$$
\lim _{T \rightarrow \infty} \phi_{T}(t)=0
$$

Next, since $\phi_{T}(s)$ is bounded and the function $s \mapsto s^{-1+\gamma(\epsilon)-\theta} e^{-a s}$ is integrable in $[0, \infty)$, using the Lebesgue dominated convergence theorem we have

$$
\lim _{T \rightarrow \infty} \frac{1}{2 T} \int_{-T}^{T}\|\Phi(t)\|_{X^{1+\theta}} d t \leq \lim _{T \rightarrow \infty} M \int_{0}^{\infty} s^{-1+\gamma(\epsilon)-\theta} e^{-a s} \phi_{T}(s) d s=0 ;
$$

therefore, $\Phi \in P_{0}\left(X^{1+\theta}\right)$, for every $0 \leq \theta<\gamma(\epsilon)$.

Step 2: Consider $u \in B(r)$, then $\|(T u)(t)\|_{X^{1+\epsilon}} \leq r$. In fact, we have

$$
\begin{aligned}
\|(T u)(t)\|_{X^{1+\epsilon}} & \leq M \int_{-\infty}^{t}(t-s)^{-1+\gamma(\epsilon)-\epsilon} e^{-a(t-s)}\|f(s, u(s))\|_{X^{\gamma}(\epsilon)} d s \\
& \leq M c \int_{-\infty}^{t}(t-s)^{-1+\gamma(\epsilon)-\epsilon} e^{-a(t-s)}\left(1+\|u(s)\|_{X^{1+\epsilon}}^{\rho}\right) d s
\end{aligned}
$$




$$
\begin{aligned}
& \leq M c \int_{-\infty}^{t}(t-s)^{-1+\gamma(\epsilon)-\epsilon} e^{-a(t-s)} d s\left(1+r^{\rho}\right) \\
& \leq M c a^{\epsilon-\gamma(\epsilon)} \Gamma(\gamma(\epsilon)-\epsilon)\left(1+r^{\rho}\right) \\
& \leq r .
\end{aligned}
$$

Now, consider $u, v \in B(r)$. We have

$$
\begin{aligned}
& \|(T u)(t)-(T v)(t)\|_{X^{1+\epsilon}} \\
& \quad \leq M \int_{-\infty}^{t}(t-s)^{-1+\gamma(\epsilon)-\epsilon} e^{-a(t-s)}\|f(s, u(s))-f(s, v(s))\|_{X^{\gamma}(\epsilon)} d s \\
& \leq M c \int_{-\infty}^{t}(t-s)^{-1+\gamma(\epsilon)-\epsilon} e^{-a(t-s)}\left(1+\|u(s)\|_{X^{1+\epsilon}}^{\rho-1}+\|v(s)\|_{X^{1+\epsilon}}^{\rho-1}\right)\|u(s)-v(s)\|_{X^{1+\epsilon}} d s \\
& \quad \leq M c\left(1+2 r^{\rho-1}\right)\left(\int_{-\infty}^{t}(t-s)^{-1+\gamma(\epsilon)-\epsilon} e^{-a(t-s)} d s\right) \sup _{t \in \mathbb{R}}\|u(t)-v(t)\|_{X^{1+\epsilon}} \\
& \leq M c a^{\epsilon-\gamma(\epsilon)} \Gamma(\gamma(\epsilon)-\epsilon)\left(1+2 r^{\rho-1}\right) \sup _{t \in \mathbb{R}}\|u(t)-v(t)\|_{X^{1+\epsilon}} .
\end{aligned}
$$

Therefore, the operator $T: B(r) \rightarrow B(r)$ is a contraction and by the Banach contraction principle we find that $T$ has a unique fixed point.

\section{Remark 3.2}

- In particular we can obtain an existence theorem in $X^{1}$ without the nonlinearity being defined on $X^{1}$. Notice that we do not assume that $f$ is a well-defined map on $X^{1}$. The only requirement on $f$ is that it is an $\epsilon$-regular map relative to $\left(X^{1}, X^{0}\right)$, for some $\epsilon>0$.

- Another important consequence of Theorem 3.1 is given when the nonlinear term $f \in \mathcal{F}(\epsilon, \gamma(\epsilon), c) \cap Z$, where

$$
Z \in\left\{\operatorname{AP}\left(X^{1+\epsilon}, X^{\gamma(\epsilon)}\right), P_{0}\left(X^{1+\epsilon}, X^{\gamma(\epsilon)}\right)\right\}
$$

In fact in this situation we can show that the operator $T: Z \rightarrow Z$ is well defined. Under the conditions of Theorem 3.1 there exists an $\epsilon$-regular mild solution $u$ of the problem (1.1). Furthermore, this solution verifies $u \in Y(1+\theta)$, for every $0 \leq \theta<\gamma(\epsilon)$, where $Y(\beta) \in\left\{\operatorname{AP}\left(X^{\beta}\right), P_{0}\left(X^{\beta}\right)\right\}$.

- An important example of our result will be the case that for a function

$$
f \in \mathcal{F}(\epsilon, \gamma(\epsilon), \rho, c) \cap \operatorname{PAP}\left(X^{1+\epsilon}, X^{\gamma(\epsilon)}\right)
$$

there exists $\lambda_{0}>0$ such that for any $0 \leq \lambda \leq \lambda_{0}$, and Theorem 3.1 applies to the function $\lambda f$.

For the sake of completeness, we include a result on globally Lipschitz maps. To this end, we consider the following class of nonlinearities: with $\epsilon, \gamma(\epsilon), \rho$, and $c$ positive constants, define

$$
\mathcal{E}(\epsilon, \gamma(\epsilon), \rho, c)
$$


as the family of functions $f$ such that, for $t \in \mathbb{R}, f(t, \cdot)$ is an $\epsilon$-regular map relative to the pair $\left(X^{1}, X^{0}\right)$, satisfying

$$
\|f(t, x)-f(t, y)\|_{X^{\gamma}(\epsilon)} \leq c\|x-y\|_{X^{1+\epsilon}}
$$

for all $x, y \in X^{1+\epsilon}$.

Theorem 3.3 Consider $f \in \mathcal{E}(\epsilon, \gamma(\epsilon), \rho, c) \cap \operatorname{PAP}\left(X^{1+\epsilon}, X^{\gamma(\epsilon)}\right)$. Suppose that

$$
M c a^{\epsilon-\gamma(\epsilon)} \Gamma(\gamma(\epsilon)-\epsilon)<1
$$

Then the problem (1.1) has a unique pseudo almost periodic $\epsilon$-regular mild solution. Furthermore, this solution verifies $u \in \operatorname{PAP}\left(X^{1+\theta}\right)$, for every $0 \leq \theta<\gamma(\epsilon)$.

Proof In fact, a similar procedure to Step 1 in Theorem 3.1 shows that the map $T$ : $\operatorname{PAP}\left(X^{1+\theta}\right) \rightarrow \operatorname{PAP}\left(X^{1+\theta}\right)$ defined by

$$
(T u)(t)=\int_{-\infty}^{t} e^{A(t-s)} f(s, u(s)) d s
$$

$t \in \mathbb{R}$, is well defined for every $0 \leq \theta<\gamma(\epsilon)$. Furthermore, if $u, v \in \operatorname{PAP}\left(X^{1+\epsilon}\right)$ then

$$
\begin{aligned}
\|(T u)(t)-(T v)(t)\|_{X \gamma(\epsilon)} & \leq M \int_{-\infty}^{t}(t-s)^{-1+\gamma(\epsilon)-\epsilon} e^{-a(t-s)}\|f(s, u(s))-f(s, v(s))\|_{X^{\gamma}(\epsilon)} d s \\
& \leq M c \int_{-\infty}^{t}(t-s)^{-1+\gamma(\epsilon)-\epsilon} e^{-a(t-s)}\|u(s)-v(s)\|_{X^{1+\epsilon}} d s \\
& \leq M c a^{\epsilon-\gamma(\epsilon)} \Gamma(\gamma(\epsilon)-\epsilon) \sup _{s \in \mathbb{R}}\|u(s)-v(s)\|_{X^{1+\epsilon}} .
\end{aligned}
$$

Therefore, the operator $T: \operatorname{PAP}\left(X^{1+\epsilon}\right) \rightarrow \operatorname{PAP}\left(X^{1+\epsilon}\right)$ is a $\left[M c a^{\epsilon-\gamma(\epsilon)} \Gamma(\gamma(\epsilon)-\epsilon)\right]$-contraction and by the Banach contraction principle $T$ has a unique fixed point.

To close this section we remember that in [24] the authors introduce a classification for a time-independent map $f$ which is $\epsilon$-regular, for $\epsilon \in I$, relative to the pair $\left(X^{1}, X^{0}\right)$. For the convenience of the reader we recall this classification.

- If $I=\left[0, \epsilon_{1}\right]$ for some $\epsilon_{1}>0$ and $\gamma(0)>0$, we say that $f$ is a subcritical map relative to $\left(X^{1}, X^{0}\right)$.

- If $I=\left[0, \epsilon_{1}\right]$ for some $\epsilon_{1}>0$ with $\gamma(\epsilon)=\rho \epsilon, \epsilon \in I$, and if $f$ is not subcritical, then we say that $f$ is a critical map relative to $\left(X^{1}, X^{0}\right)$.

- If $I=\left(0, \epsilon_{1}\right]$ for some $\epsilon_{1}>0$ with $\gamma(\epsilon)=\rho \epsilon, \epsilon \in I$, and $f$ is not subcritical or critical, then we say that $f$ is a double critical map relative to $\left(X^{1}, X^{0}\right)$.

- If $I=\left[\epsilon_{0}, \epsilon_{1}\right]$ for some $\epsilon_{1}>\epsilon_{0}>0$ with $\gamma\left(\epsilon_{0}\right)>\rho \epsilon_{0}$ and $f$ is not subcritical, critical or double critical, then we say that $f$ is an ultra-subcritical map relative to $\left(X^{1}, X^{0}\right)$.

- If $I=\left[\epsilon_{0}, \epsilon_{1}\right]$ for some $\epsilon_{1}>\epsilon_{0}>0$ with $\gamma(\epsilon)=\rho \epsilon, \epsilon \in I$, and if $f$ is not subcritical, critical, double critical or ultra-subcritical, then we say that $f$ is an ultra-critical map relative to $\left(X^{1}, X^{0}\right)$. 


\section{Applications to heat equation}

Let $\Omega \subset \mathbb{R}^{N}$ be a bounded smooth domain. In this section we will treat the equation

$$
\begin{cases}u_{t}=A u+g h(u), & \text { in } \mathbb{R} \times \Omega, \\ u=0, & \text { on } \mathbb{R} \times \partial \Omega,\end{cases}
$$

where $g \in \operatorname{PAP}(\mathbb{R}), A u=\Delta u-a u, a>0$, and the function $h: \mathbb{R} \rightarrow \mathbb{R}$ verifies

$$
|h(x)-h(y)| \leq c\left(1+|x|^{\rho-1}+|y|^{\rho-1}\right)|x-y|, \quad \rho>1,
$$

for some $c>0$. It is well known that there are many works on equations like (4.1). In particular, we suggest to the reader the references [22-24,29]. We are specially interested in the $L^{q}$ theory, $1<q<\infty$ and $q=\frac{N(\rho-1)}{2}$.

Let $L=\Delta$ with Dirichlet boundary conditions in $\Omega$. Then $L$ can be seen as an unbounded operator in $E_{q}^{0}=L^{q}(\Omega)$ with domain

$$
D(A):=W^{2, q}(\Omega) \cap W_{0}^{1, q}(\Omega) .
$$

It is well known that the scale of fractional power spaces $\left\{E_{q}^{\alpha}\right\}_{\in \in \mathbb{R}}$ associated to $L$ verifies (see $[24,26])$

$$
\begin{aligned}
& E_{q}^{\alpha} \hookrightarrow H_{q}^{2 \alpha}(\Omega), \quad \alpha \geq 0,1<q<\infty, \\
& E_{q}^{-\alpha} \hookrightarrow\left(E_{q^{\prime}}^{\alpha}\right)^{\prime}, \quad \alpha \geq 0,1<q<\infty, q^{\prime}=\frac{q}{q-1} .
\end{aligned}
$$

Therefore, we get

$$
\begin{aligned}
& E_{q}^{\alpha} \hookrightarrow L^{r}(\Omega), \quad \text { for } r \leq \frac{N q}{N-2 q \alpha}, 0 \leq \alpha \leq \frac{N}{2 q}, \\
& E_{q}^{0}=L^{q}(\Omega), \\
& E_{q}^{\alpha} \hookleftarrow L^{r}(\Omega), \quad \text { for } r \geq \frac{N}{N-2 q \alpha},-\frac{N}{2 q^{\prime}} \leq \alpha \leq 0 .
\end{aligned}
$$

Let $L_{\alpha}$ be the realization of $L$ in $E_{q}^{\alpha}$. Then we find that $L_{\alpha}$ is an isometry from $E_{q}^{\alpha+1}$ into $E_{q}^{\alpha}$ and

$$
L_{\alpha}: D\left(L_{\alpha}\right)=E_{q}^{\alpha+1} \subset E_{q}^{\alpha} \rightarrow E_{q}^{\alpha}
$$

is a sectorial operator. Denote $X_{q}^{\alpha}:=E_{q}^{\alpha-1}, \alpha \in \mathbb{R}$. The fractional power spaces associated to $L_{-1}: X_{q}^{1} \subset X_{q}^{0} \rightarrow X_{q}^{0}$ satisfy

$$
\begin{aligned}
& X_{q}^{\alpha} \hookrightarrow L^{r}(\Omega), \quad \text { for } r \leq \frac{N q}{N+2 q-2 q \alpha}, 1 \leq \alpha<\frac{2 q+N}{2 q}, \\
& X_{q}^{1}=L^{q}(\Omega), \\
& X_{q}^{\alpha} \hookleftarrow L^{r}(\Omega), \quad \text { for } r \geq \frac{N q}{N+2 q-2 q \alpha}, \frac{2 q^{\prime}-N}{2 q^{\prime}}<\alpha \leq 1 .
\end{aligned}
$$


Consider $f: \mathbb{R} \times \mathbb{R} \rightarrow \mathbb{R}$ defined by

$$
f(t, x)=g(t) h(x)
$$

We can to classify this function in the following way (see [24, Lemma 8]):

- If $q>\frac{N}{N-2}$, then $f$ is an $\epsilon$-regular map relative to $\left(X_{q}^{1}, X_{q}^{0}\right)$ for $0=\epsilon_{0}(q) \leq \epsilon<\frac{N}{N+2 q}$ and $\gamma(\epsilon)=\rho \epsilon$. Therefore $f$ is a critical map.

- If $q=\frac{N}{N-2}$, then $f$ is an $\epsilon$-regular map relative to $\left(X_{q}^{1}, X_{q}^{0}\right)$ for $0=\epsilon_{0}(q)<\epsilon<\frac{N}{N+2 q}$ and $\gamma(\epsilon)=\rho \epsilon$. Therefore $f$ is a double critical map.

- If $1<q<\frac{N}{N-2}$, then $f$ is an $\epsilon$-regular map relative to $\left(X_{q}^{1}, X_{q}^{0}\right)$ for $0<\epsilon_{0}(q)<\epsilon<\frac{N}{N+2 q}$, with $\epsilon_{0}(q)=\frac{N}{N+2 q}\left(1-\frac{N}{2}\left(1-\frac{1}{q}\right)\right)>0$, and $\gamma(\epsilon)=\rho \epsilon$. Therefore $f$ is an ultra-critical map. A straightforward computation shows that

$$
f \in \mathcal{F}(\epsilon, \gamma(\epsilon), \rho, c) \cap \operatorname{PAP}\left(X^{1+\epsilon}, X^{\gamma(\epsilon)}\right)
$$

for any $\epsilon \in\left(\epsilon_{0}(q), \frac{N}{N+2 q}\right)$. If the constants $M$ and $c$ are as in Theorem 3.1 then there exists a mild solution $u \in \operatorname{PAP}\left(X^{1+\epsilon}\right)$ of (4.1) such that $u \in \operatorname{PAP}\left(X^{1+\theta}\right)$, for every $0 \leq \theta<\gamma(\epsilon)$.

\section{Conclusions}

We have studied the existence of pseudo almost periodic mild solutions for abstract evolution equation when the nonlinearity satisfies certain critical conditions. Investigations in such directions are technically more complicated. The difficulty when we have the critical growth relies on the fact that the nonlinear terms are 'too large' and cannot be controlled a priori by the linear terms. Therefore, for this case, the standard approach to semilinear equations, e.g., [26, 28], fails (see also [25]). To achieve our results we use the scale of fractional power spaces and $\epsilon$-regularity theory developed by Arrieta and Carvalho in [24] (this paper was very inspiring for us). Note that problems with this kind of nonlinearity appear frequently in the heat equation theory. We have applied our abstract results to such equations. Our results are new and contribute to the development of the asymptotic periodicity theory of evolution equations.

Competing interests

The authors declare that they have no competing interest.

Authors' contributions

Each of the authors contributed to each part of this study equally and approved the final version of this manuscript.

\section{Author details}

'Departamento de Matemática, Universidade Federal de Sergipe, São Cristóvão, SE 49100-000, Brazil. ²Departamento de Matemática, Universidade Federal de Pernambuco, Recife, PE 50540-740, Brazil. ${ }^{3}$ Department of Mathematics, Shanghai Jiao Tong University, Shanghai, 200240, People's Republic of China. ${ }^{4}$ Departamento de Matemática y Estadística, Universidad de La Frontera, Casilla 54D, Temuco, Chile.

\section{Acknowledgements}

B de Andrade is partially supported by CNPQ/Brazil under Grant 100994/2011-3. C Cuevas is partially supported by CNPQ/Brazil under Grant 478053/2013-4. H Soto is partially supported by DIUFRO (Universidad de La Frontera) under Grant DI15-0050.

Received: 7 October 2014 Accepted: 25 December 2014 Published online: 30 January 2015

\section{References}

1. Henríquez, H, Cuevas, C, Caicedo, A: Asymptotically periodic solutions of neutral partial differential equations with infinite delay. Commun. Pure Appl. Anal. 12(5), 2031-2068 (2013) 
2. Batty, CJK, Hutter, W, Räbiger, F: Almost periodicity of mild solutions of inhomogeneous periodic Cauchy problems. J. Differ. Equ. 156, 309-327 (1999)

3. Ruess, WM, Vu, QP: Asymptotically almost periodic solutions of evolution equations in Banach spaces. J. Differ. Equ. $122,282-301(1995)$

4. Agarwal, RP, de Andrade, B, Cuevas, C: Weighted pseudo-almost periodic solutions of a class of semilinear fractional differential equations. Nonlinear Anal., Real World Appl. 11, 3532-3554 (2010)

5. Cuevas, C, Pinto, M: Existence and uniqueness of pseudo almost periodic solutions of semilinear Cauchy problems with non dense domain. Nonlinear Anal. 45, 73-83 (2001)

6. Cuevas, C, Hernández, H: Pseudo almost periodic solutions for abstract partial functional differential equations. Appl. Math. Lett. 22, 534-538 (2009)

7. Diagana, T: Weighted pseudo almost periodic functions and applications. C. R. Math. Acad. Sci. Paris 343(10), 643-646 (2006)

8. Diagana, T: Pseudo Almost Periodic Functions in Banach Spaces. Nova Science Publishers, New York (2007)

9. Diagana, T: Existence of weighted pseudo almost periodic solutions to some classes of hyperbolic evolution equations. J. Math. Anal. Appl. 350, 18-28 (2009)

10. Ding, HS, Liang, J, Xiao, TJ: Pseudo almost periodic solutions to integro-differential equations of heat conduction in materials with memory. Nonlinear Anal., Real World Appl. 13, 2659-2670 (2012)

11. Ding, HS, Liang, J, N'Guérékata, GM, Xiao, TJ: Pseudo-almost periodicity of some nonautonomous evolution equations with delay. Nonlinear Anal. 67, 1412-1418 (2007)

12. Liang, J, Xiao, TJ, Zhang, J: Decomposition of weighted pseudo-almost periodic functions. Nonlinear Anal. 73 , 3456-3461 (2010)

13. Liu, JH, Song, XQ: Almost automorphic and weighted pseudo almost automorphic solutions of semilinear evolution equations. J. Funct. Anal. 258, 196-207 (2010)

14. Zhang, J, Xiao, TJ, Liang, J: Weighted pseudo almost-periodic functions and applications to semilinear evolution equations. Abstr. Appl. Anal. 2012, Article ID 179525 (2012)

15. Zhang, C: Pseudo almost periodic solutions of some differential equations. J. Math. Anal. Appl. 151, $62-76$ (1994)

16. Zhang, C: Integration of vector-valued pseudo almost periodic functions. Proc. Am. Math. Soc. 121, 167-174 (1994)

17. Zhang, C: Pseudo almost periodic solutions of some differential equations II. J. Math. Anal. Appl. 192, 543-561 (1995)

18. Zhang, C: Almost Periodic Type and Ergodicity. Kluwer Academic, Dordrecht (Science Press, Beijing) (2003)

19. Ashyralyev, A, Sozen, Y, Sobolevskii, PE: A note on the parabolic differential and difference equations. Abstr. Appl. Anal. 2007, Article ID 61659 (2007)

20. Ashyralyev, A, Sozen, Y: A note on the parabolic equation with an arbitrary parameter at the derivative. Math. Comput. Model. 54(11-12), 2565-2572 (2011)

21. Ashyralyev, A, Sozen, Y: Well-posedness of parabolic differential and difference equations. Comput. Math. Appl. 60(3), 792-802 (2010)

22. Weissler, FB: Semilinear evolution equations in Banach spaces. J. Funct. Anal. 32, 277-296 (1979)

23. Weissler, FB: Local existence and nonexistence for semilinear parabolic equations in $L^{p}$. Indiana Univ. Math. J. 29, 79-102 (1980)

24. Arrieta, JM, Carvalho, AN: Abstract parabolic problems with critical nonlinearities and applications to Navier-Stokes and heat equations. Trans. Am. Math. Soc. 352, 285-310 (2000)

25. Arrieta, JM, Carvalho, AN, Rodríguez-Bernal, A: Parabolic problems with nonlinear boundary conditions and critical nonlinearities. J. Differ. Equ. 156, 376-406 (1999)

26. Amann, H: Nonhomogeneous linear and quasilinear elliptic and parabolic boundary value problems. In: Schmeisser, H-J, Triebel, H (eds.) Function Spaces, Differential Operators and Nonlinear Analysis. Teubner Texte zur Mathematik, vol. 133, pp. 9-126. Teubner, Leipzig (1993)

27. Fink, AM: Almost Periodic Differential Equations. Lectures Notes in Mathematics, vol. 377. Springer, Berlin (1974)

28. Henry, D: Geometric Theory of Semilinear Parabolic Equations. Lectures Notes in Mathematics, vol. 840. Springer, Berlin (1981)

29. Brezis, H, Cazenave, T: A nonlinear heat equation with singular initial data. J. Anal. Math. 68, 277-304 (1996)

\section{Submit your manuscript to a SpringerOpen ${ }^{\circ}$ journal and benefit from:}

- Convenient online submission

Rigorous peer review

- Immediate publication on acceptance

- Open access: articles freely available online

- High visibility within the field

- Retaining the copyright to your article 